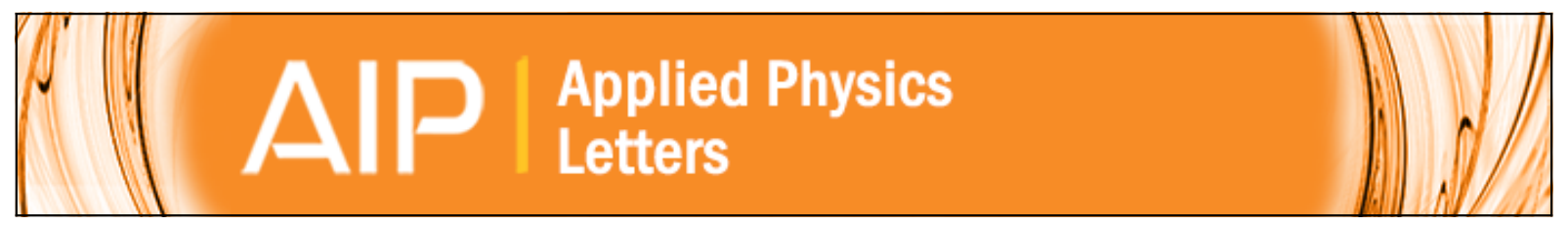

Femtosecond nonlinear optical properties of lead-germanium oxide amorphous films

Diego Rativa, Renato E. de Araujo, Cid B. de Araújo, Anderson S. L. Gomes, and Luciana R. P. Kassab

Citation: Applied Physics Letters 90, 231906 (2007); doi: 10.1063/1.2747174

View online: http://dx.doi.org/10.1063/1.2747174

View Table of Contents: http://scitation.aip.org/content/aip/journal/apl/90/23?ver=pdfcov

Published by the AIP Publishing 


\title{
Femtosecond nonlinear optical properties of lead-germanium oxide amorphous films
}

\author{
Diego Rativa and Renato E. de Araujo \\ Departamento de Engenharia Eletrônica e Sistemas, Universidade Federal de Pernambuco, \\ 50740-530 Recife, Pernambuco, Brazil \\ Cid B. de Araújo ${ }^{\text {a) }}$ and Anderson S. L. Gomes \\ Departamento de Física, Universidade Federal de Pernambuco, 50670-901 Recife, Pernambuco, Brazil \\ Luciana R. P. Kassab \\ Laboratório de Vidros e Datação, Faculdade de Tecnologia de São Paulo, CEETEPS/UNESP, \\ 01124-060 São Paulo, Sã Paulo, Brazil
}

(Received 21 March 2007; accepted 14 May 2007; published online 6 June 2007)

\begin{abstract}
The nonlinear (NL) response of lead-germanium oxide amorphous films was investigated using a Ti:saphire laser delivering pulses of $\approx 150$ fs at $800 \mathrm{~nm}$. The Kerr shutter technique was employed to reveal the time response of the nonlinearity that is smaller than $150 \mathrm{fs}$. The sign and magnitude of the nonlinearity were obtained using a novel technique called thermally managed eclipse $\mathrm{Z}$ scan which allows the simultaneous characterization of cumulative and noncumulative NL effects. The NL refractive index of electronic origin, $n_{2} \approx 2 \times 10^{-17} \mathrm{~m}^{2} / \mathrm{W}$, and the NL absorption coefficient, $\alpha_{2} \approx 3 \times 10^{3} \mathrm{~cm} / \mathrm{GW}$, were determined. (C) 2007 American Institute of Physics.
\end{abstract}

[DOI: $10.1063 / 1.2747174]$

The search of new materials with large nonlinearity is motivated by the development of devices for optical processing, all-optical switching and optical limiting, among other applications. Several families of heavy-metal oxide (HMO) glasses that are promising candidates for such photonic devices have been reported. ${ }^{1-10}$ In particular, compositions of HMO glasses containing lead and/or bismuth are strong candidates and have been studied in the past few years. ${ }^{6-10}$ Recent measurements with bismuth oxide based glasses from the femtosecond to the nanosecond regime ${ }^{6,7}$ demonstrated that the presence of heavy-metal atoms is very important to enhance the nonlinearity. Previous studies using pulses of 35 and $100 \mathrm{fs}$ at wavelengths in the range of $600-1250 \mathrm{~nm}$ also revealed increasing of the HMO glasses nonlinearity with the increase of heavy-metal content. ${ }^{8}$ Measurements of fifth- and seventh-order nonlinearities of some HMO glasses were performed at $790 \mathrm{~nm}$ with pulses of $100 \mathrm{fs} .{ }^{9}$

Among the HMO families, the lead-germanate glasses deserve a lot of attention because they are simple to prepare, have high refractive indices $(\approx 2)$, present large transmission in the visible and in the near infrared, are very stable and resistant to moisture, and have small cutoff phonon energy $\left(\approx 700 \mathrm{~cm}^{-1}\right)$. For instance, experiments performed with laser pulses of $15 \mathrm{ps}$ at $1064 \mathrm{~nm}$ have shown that HMO glasses based on $\mathrm{PbO}-\mathrm{GeO}_{2}$ present large nonlinear (NL) refractive index, $n_{2} \approx 10^{-18} \mathrm{~m}^{2} / \mathrm{W}$, negligible NL absorption coefficient $\alpha_{2}$, and good figure of merit for all-optical switching applications. ${ }^{10}$ More recently large efficiency of second harmonic generation in $\mathrm{Er}^{3+}$ doped $\mathrm{PbO}-\mathrm{GeO}_{2}$ induced by two-color optical poling was obtained. ${ }^{11}$

Although films containing heavy-metal constituents also attract a great deal of interest ${ }^{1,12-14}$ the femtosecond NL properties of amorphous films of lead-germanium oxides

a) Author to whom correspondence should be addressed; electronic mail: cid@df.ufpe.br have not been investigated yet. Recently, the nonlinearity of lead-germanium based films (LGFs) was studied using a $15 \mathrm{ps}$ neodymium doped yttrum aluminum garnet laser at $1064 \mathrm{~nm}$ and its second harmonic at $532 \mathrm{~nm} .{ }^{15} \mathrm{NL}$ refractive indices of $\approx 10^{-16} \mathrm{~m}^{2} / \mathrm{W}$ and NL absorption coefficient varying from $\approx 10^{2} \mathrm{~cm} / \mathrm{GW}$ at $1064 \mathrm{~nm}$ to $\approx 10^{3} \mathrm{~cm} / \mathrm{GW}$ at $532 \mathrm{~nm}$ were measured.

In this letter, we report on the LGF nonlinearity in the femtosecond regime as well as present measurements of $n_{2}$ and $\alpha_{2}$ that show large nonlinearity for excitation at $800 \mathrm{~nm}$. The experiments were made using the Kerr shutter technique $^{16}$ and the thermally managed eclipse Z-scan (TM-EZ scan) technique. ${ }^{17}$ Large values of $n_{2} \approx 2 \times 10^{-17} \mathrm{~m}^{2} / \mathrm{W}$ and $\alpha_{2} \approx 3 \times 10^{3} \mathrm{~cm} / \mathrm{GW}$ were measured. Carbon disulfide $\left(\mathrm{CS}_{2}\right)$ was used as a reference material to confirm our data.

Films with a thickness of $1.5 \mu \mathrm{m}$ were fabricated on quartz substrates using the rf sputtering method $(50 \mathrm{~W}$, $14 \mathrm{MHz}$ ). Pure argon plasma was used at a constant pressure of 5.5 mTorr. The glassy targets were obtained by melting the starting materials in an alumina crucible at $1050{ }^{\circ} \mathrm{C}$ for $1 \mathrm{~h}$, quenched in air, in a heated graphite mold, annealed for $1 \mathrm{~h}$ at $420{ }^{\circ} \mathrm{C}$, and then cooled to room temperature inside a furnace. The films obtained exhibit good optical quality, high mechanical strength, and large adherence to quartz substrate.

For the NL experiments we used a Ti-sapphire laser $(800 \mathrm{~nm}, 150 \mathrm{fs}, 76 \mathrm{MHz})$. The Kerr shutter setup is well known. ${ }^{16}$ The laser beam is split into two beams with different intensities. The electric field of the strong (pump) beam is set at $45^{\circ}$ with respect to the electric field of the weak (probe) input beam. When the pulses of both beams overlap spatially and temporally at the sample position, the probe beam polarization rotates due to the birefringence induced in the sample by the pump beam. Then, a fraction of the probe beam passes through a polarizer crossed to the input probe beam polarization. A slow detector is used to record the 


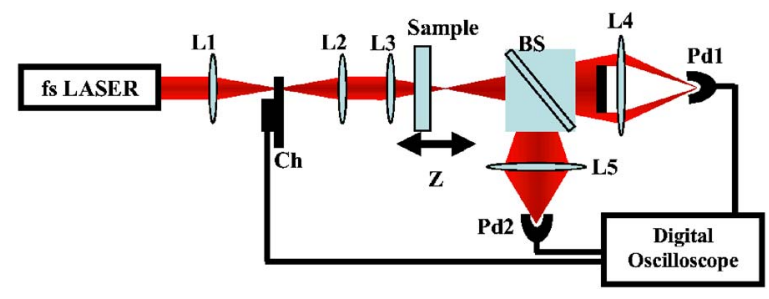

FIG. 1. Experimental setup: L1-L5 are biconvex lenses. BS is a beam splitter. Pd1 and Pd2 are photodiodes. Ch is a chopper.

probe signal as a function of the delay between the pump and the probe pulses.

The TM-EZ scan technique is a combination of the eclipse $\mathrm{Z}$ scan ${ }^{18}$ with the thermally managed $\mathrm{Z}$ scan. ${ }^{19}$ The new technique was introduced recently for studies of liquids, solids, and biomaterials. ${ }^{7,17}$ TM-EZ scan technique presents the sensitivity of eclipse $\mathrm{Z}$ scan and gives the simultaneous measurements of the nonthermal and thermal nonlinearities of the material under study. The use of lasers with high repetition rate allows measurements with large sensitivity and better signal-to-noise figure.

The experimental setup for TM-EZ scan, shown in Fig. 1, employs a disk in front of the detector such that the eclipsed beam collected by a lens is directed towards the detector. For large disk and small NL phase shift $\left(\Delta \Phi_{0}<0.2\right)$ the relationship between $\Delta T_{p v}$ and $\Delta \Phi_{0}$ can be written as $\Delta T_{p v}=0.68\left(1-S_{d}\right)^{-0.44}\left|\Delta \Phi_{0}\right|$, where $S_{d}$ is the fraction of the beam blocked by the disk, given by $S_{d}=\left[1-\exp \left(-2 r_{d}^{2} / w_{d}^{2}\right)\right]$, with $r_{d}$ being the disk radius and $w_{d}$ the beam radius at the disk position. The NL phase shift is given by $\Delta \Phi_{0}=k n_{2} I_{0} L_{\text {eff }}$ where $I_{0}$ is the excitation peak intensity within the sample, $k=2 \pi / \lambda, \quad L_{\text {eff }}$ $=\left[1-\exp \left(-\alpha_{o} L\right)\right] / \alpha_{o}$, and $L$ is the sample length. The chopper is the new element responsible for the thermal or other cumulative effects management, introduced to modify the conventional EZ-scan setup. In short, the TM-EZ scan method consists in acquiring the time evolution of the EZscan signal, for the sample placed in the pre- and postfocal positions of its focal plane with respect to lens L3. The time resolution of the system is determined by the chopper opening time $\left(\tau_{o}=10 \mu \mathrm{s}\right.$ in our setup), which depends on the finite size of the beam waist on the chopper wheel. By extrapolating the time evolution curves for $t<\tau_{o}$, noncumulative signals at both the pre- and postfocal positions are obtained. The photodetector information is sent to a digital scope and then processed. From these measurements, using the formalism described in Ref. 20, the EZ-scan curves can be constructed and the contribution of cumulative effects (such as thermal effects) and electronic nonlinearities can be inferred, provided no other mechanism besides the electronic nonlinearity are present in the relatively short time of the chopper opening rise time. The technique is sensitive to $\mathrm{cu}-$ mulative effects such as thermal effect and contributions due to absorption of excited carriers from states of long depopulation time.

Figure 2 shows the Kerr shutter results obtained for $\mathrm{CS}_{2}$ and for the LGF sample. As is well known, $\mathrm{CS}_{2}$ has two decay times, a fast one $(<50 \mathrm{fs})$ and a slower one $(\sim 2 \mathrm{ps})$. On the other hand, the signal due to the LGF is symmetric. The inset in Fig. 2 shows the result in detail to illustrate the fast behavior of the signal, demonstrating that the sample response is iimited by the pulse duration ( $150 \mathrm{fs})$. The power

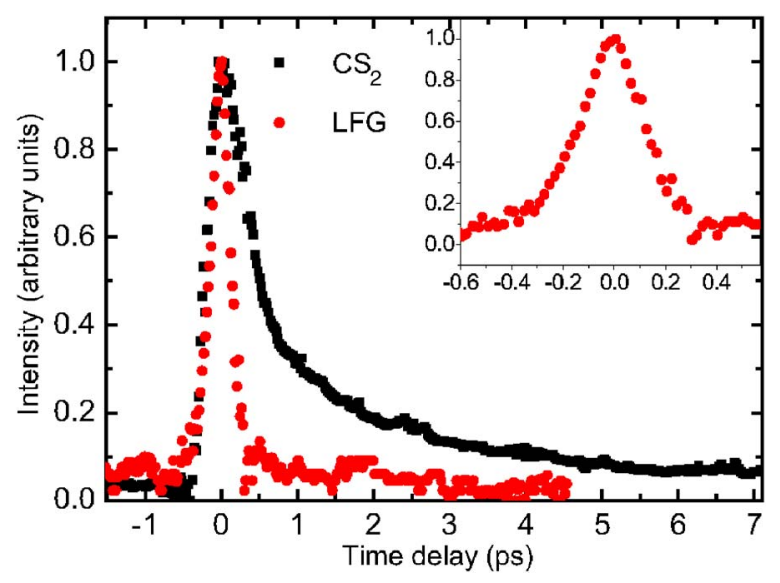

FIG. 2. Normalized Kerr shutter signals for $\mathrm{CS}_{2}$ (solid squares) and the lead-germanium film (solid circles). The inset shows the Kerr shutter signal for the film in an expanded scale.

dependence of the Kerr signal intensity versus pump power indicated a dependence of the signal with the square of pump beam intensity $\left(I_{\text {signal }} \propto I_{\text {probe }} I_{\text {pump }}^{2}\right)$, which arises due to the phase shift imposed on the probe beam by the pump beam.

Figures 3 and 4 show the results of the TM-EZ scan experiments. The solid lines are the best-fit curves obtained using the procedure described in Ref. 20. For the sake of comparison, as well as intensity calibration, we first performed measurements for liquid $\mathrm{CS}_{2}$ contained in a cell of $2 \mathrm{~mm}$. For measurements of the signal temporal evolution the cell is placed in the peak and valley transmittance posi-
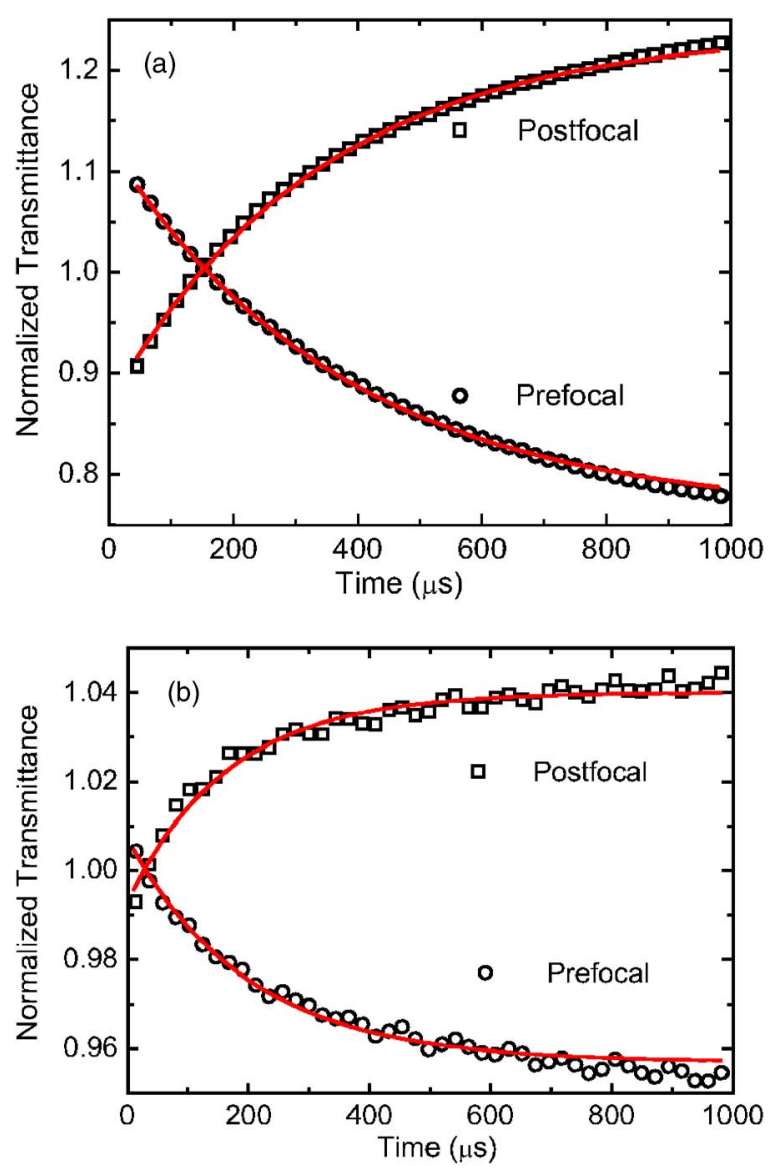

FIG. 3. Time evolution of the TM EZ-scan signal at pre- and postfocal positions. (a) Liquid $\mathrm{CS}_{2}$. (b) Lead-germanium film. 

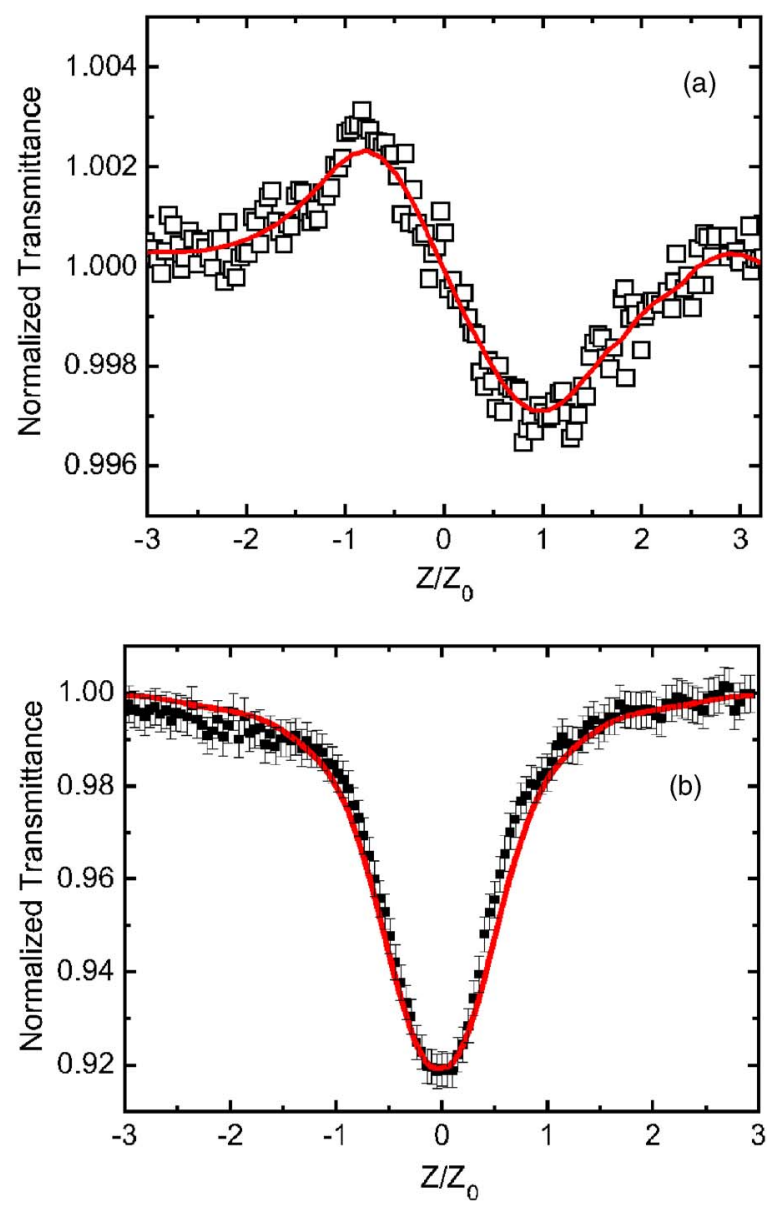

FIG. 4. TM-EZ scan profile for the lead-germanium film. (a) NL refractive signal $\left(S_{d}=0.99\right)$. (b) NL absorption signal $\left(S_{d}=1\right)$.

tion and the results are recorded for different delay times. The crossing of the two temporal evolution curves obtained at the pre- and postfocal positions indicates the presence of both cumulative and noncumulative nonlinearities. By extrapolating the time evolution curves of $\mathrm{CS}_{2}$ for $t=0$, the normalized peak-valley transmittance, for $S_{d}=0.99$, is $\Delta T_{p v}=0.25$, corresponding to $1.6 \mathrm{GW} / \mathrm{cm}^{2}$ at the focus. The curves cross at $\tau_{c} \approx 150 \mu \mathrm{s}$, indicating the dominance of the thermal nonlinearity on $\mathrm{CS}_{2}$ after the crossing. Figure 3(b) shows the result for the LGF sample. Lower light intensity $\left(0.5 \mathrm{GW} / \mathrm{cm}^{2}\right)$ was used to prevent damage of the sample. In this case the temporal evolution curves cross at $\tau_{c} \approx 30 \mu \mathrm{s}$. By extrapolating the curves to $t=0$ the total change in the normalized transmittance is $\approx 0.005$. From the measurements of Fig. 3(b) we obtain $n_{2}=2 \pm 1 \times 10^{-17} \mathrm{~m}^{2} / \mathrm{W}$. This result represents the average of eight measurements at different sample regions. A typical signal profile obtained for $t<\tau_{\text {cross }}$ is shown in Fig. 4(a) where the solid line represents the theoretical fitting. NL absorption was also measured and Fig. 4(b) shows a typical result corresponding to $\alpha_{2}=(3 \pm 1) \times 10^{3} \mathrm{~cm} / \mathrm{GW}$.

To analyze the present measurements we first recall the previous results obtained using a $15 \mathrm{ps}$ laser at $1064 \mathrm{~nm}$ and its second harmonic at $532 \mathrm{~nm} .{ }^{15}$ In that experiment $n_{2}$ and $\alpha_{2}$ did not change when the laser intensity was increased by almost one order of magnitude, indicating that high-order nonlinearities were not present. In the present experiment $n_{2}$ is smaller than that for 532 and $1064 \mathrm{~nm}$ by about one order of magnitude; $\alpha_{2}$ at $800 \mathrm{~nm}$ has the same order of magnitude than that at $532 \mathrm{~nm}$ and is one order of magnitude larger than that at $1064 \mathrm{~nm}$. The increase of $\alpha_{2}$ is attributed to energy states located inside the energy gap due to possible microscopic defects in the film. The localized states may present long relaxation time ${ }^{1}$ and originate a tail in the absorption spectrum of the LGF such that the linear absorption coefficient at $1064 \mathrm{~nm}$ is $6.7 \times 10^{2} \mathrm{~cm}^{-1}$ and its value at $800 \mathrm{~nm}$ is $7.2 \times 10^{2} \mathrm{~cm}^{-1}$. It is probable that the value of $\alpha_{2}$ is enhanced through resonance with intermediate states. Of course, the localized states may also originate cumulative effects that would increase the value of $n_{2}$, but the value measured using the TM-EZ scan technique is of pure electronic origin and it is not affected by cumulative effects.

The large values obtained for $n_{2}$ and $\alpha_{2}$ indicate that the LGF herein studied can be used as optical limiter for laser pulses of $150 \mathrm{fs}$.

Financial support by the Brazilian agencies Conselho Nacional de Desenvolvimento Científico e Tecnológico (CNPq) and Fundação de Amparo a Ciência e Tecnologia do Estado de Pernambuco (FACEPE) is acknowledged. This work was performed under the Millenium Institute on Nonlinear Optics, Photonics and Bio-Photonics Project and the Nanophotonics Network Program. The Instituto Tecnológico da Aeronáutica is also acknowledged for the sputtering equipment used for the film production.

${ }^{1}$ See, for instance, M. Yamane and Y. Asahara, Glasses for Photonic (Cambridge University Press, Cambridge, UK, 2000).

${ }^{2}$ K. Tanaka, J. Mater. Sci.: Mater. Electron. 16, 633 (2005).

${ }^{3}$ T. Hashimoto, T. Yamamoto, T. Kato, H. Nasu, and K. Kamiya, J. Appl. Phys. 90, 533 (2001).

${ }^{4}$ E. L. Falcão-Filho, C. B. de Araújo, C. A. C. Bosco, G. S. Maciel, L. H. Acioli, M. Nalin, and Y. Messaddeq, J. Appl. Phys. 97, 013505 (2005).

${ }^{5}$ L. A. Gomez, C. B. de Araújo, D. N. Messias, L. Misoguti, S. C. Zílio, M. Nalin, and Y. Messaddeq, J. Appl. Phys. 100, 116105 (2006).

${ }^{6}$ T. R. Oliveira, L. de S. Menezes, E. L. Falcão-Filho, A. S. L. Gomes, C. B. de Araújo, K. Sakaguchi, F. P. Mezzapesa, I. C. S. Carvalho, and P. G. Kazansky, Appl. Phys. Lett. 89, 21912 (2006).

${ }^{7}$ A. S. L. Gomes, E. L. Falcão-Filho, C. B. de Araújo, D. Rativa, R. E. de Araujo, K. Sakaguchi, F. P. Mezzapesa, I. C. S. Carvalho, and P. G. Kazansky, J. Appl. Phys. 101, 033115 (2007).

${ }^{8}$ S. Smolorz, I. Kang, F. Wise, B. G. Aitken, and N. F. Borrelli, J. Non-Cryst. Solids 256, 310 (1999).

${ }^{9}$ Y. F. Chen, K. Beckwitt, F. Wise, B. G. Aitken, J. S. Sanghera, and I. D. Aggarwal, J. Opt. Soc. Am. B 23, 347 (2006).

${ }^{10}$ C. B. de Araújo, E. L. Falcão-Filho, A. Humeau, D. Guichaoua, G. Boudebs, and L. R. P. Kassab, Appl. Phys. Lett. 87, 221904 (2005).

${ }^{11}$ L. R. P. Kassab, W. G. Hora, M. Piasecki, P. Bragiel, and I. V. Kityk, Opt. Commun. 269, 148 (2007).

${ }^{12}$ Y. Z. Gu, W. F. Zhang, D. H. Gu, and F. X. Gan, Opt. Lett. 26, 1788 (2001).

${ }^{13}$ B. Gu, Y. H. Wang, X. C. Peng, J. P. Ding, J. L. He, and H. T. Wang, Appl. Phys. Lett. 85, 3687 (2004).

${ }^{14}$ O. Sanz, J. Gonzalo, A. Perea, J. M. Fernández-Navarro, C. N. Afonso, and J. Garcia López, Appl. Phys. A: Mater. Sci. Process. 79, 1907 (2004).

${ }^{15}$ C. B. de Araújo, A. Humeau, G. Boudebs, V. D. Del Cacho, and L. R. P. Kassab, J. Appl. Phys. 101, 066103 (2007).

${ }^{16}$ R. L. Sutherland, Handbook of Nonlinear Optics (Wiley, New York, 1996).

${ }^{17}$ A. S. L. Gomes, E. L. Falcão-Filho, C. B. de Araújo, D. Rativa, and R. E. de Araujo, Opt. Express 15, 1712 (2007).

${ }^{18}$ T. Xia, D. J. Hagan, M. Sheik-Bahae, and E. W. van Stryland, Opt. Lett. 19, 317 (1994).

${ }^{19}$ A. Gnoli, L. Razzari, and M. Righini, Opt. Express 13, 7976 (2005).

${ }^{20}$ M. Sheik-Bahae, A. A. Said, T. Wei, D. J. Hagan, and E. W. van Stryland, IEEE J. Quantum Electron. 26, 760 (1990). 